\title{
Evaluation of Response Spectrum Considering the Stochastic Characteristics of the Phase Spectrum of Earthquake Motion
}

\author{
Yoshitaka MURONO, Dr. Eng. \\ Earthquake and Structural Engineering Laboratory, \\ Structures Technology Division
}

\begin{abstract}
It is well known that the non-stationary nature of earthquake motion is intensely controlled by the phase spectrum. However, it has not yet been verified how the stochastic characteristics of phase spectrum of such motion affects the response of structures. We therefore modeled the stochastic characteristics of the phase spectrum, and derived a theoretical solution for mean and R.M.S. response time histories for an SDOF system excited by a non-stationary input ground motion controlled by these stochastic phase characteristics. We also derived a methodology to estimate the peak response value using the concept of a complex envelope function.
\end{abstract}

Keywords: phase spectrum, stochastic characteristic, complex envelope function, SDOF system

\section{Introduction}

The expected peak value for a stationary random process can be obtained using the Spectrum Moment Method [1], which requires only the amplitude spectrum to derive the peak response of a structure. If earthquake motion were a stationary random process, therefore, the peak response could be readily derived using only the Fourier amplitude spectrum of the input earthquake motion and the frequency response function of the structure. However, earthquake ground motion is obviously a non-stationary process, and its nature is intensely controlled by its phase characteristics [2]. This suggests that it is necessary to formulate structural response statistically by taking into account the probabilistic nature of the phase spectrum to obtain the expected peak response value of structures. A methodology to introduce the stochastic characteristics of the phase spectrum into random vibration analysis of a SDOF (single-degree-offreedom) system has been developed, and close solutions of the mean and variance of structural response are derived in this paper. We also conducted numerical simulations using this formula.

\section{Stochastic characteristics of phase spectrum}

\subsection{Definition of group delay time}

The concept of group delay time $t_{g r}(\omega)$ is used to model a phase spectrum $\phi(\omega)$. The group delay time $t_{g r}(\omega)$ is defined by a derivative of the Fourier phase spectrum $\phi(\omega)$ with respect to circular frequency $\omega[3]$ :

$$
t_{g r}(\omega)=\frac{d \phi(\omega)}{d \omega}
$$

Figure 1 shows an example of group delay time calculated for earthquake ground motion and band-passed waves. It is evident from the figure that the average group delay time within a certain frequency band with a central frequency of $\omega$ expresses the arrival time of a
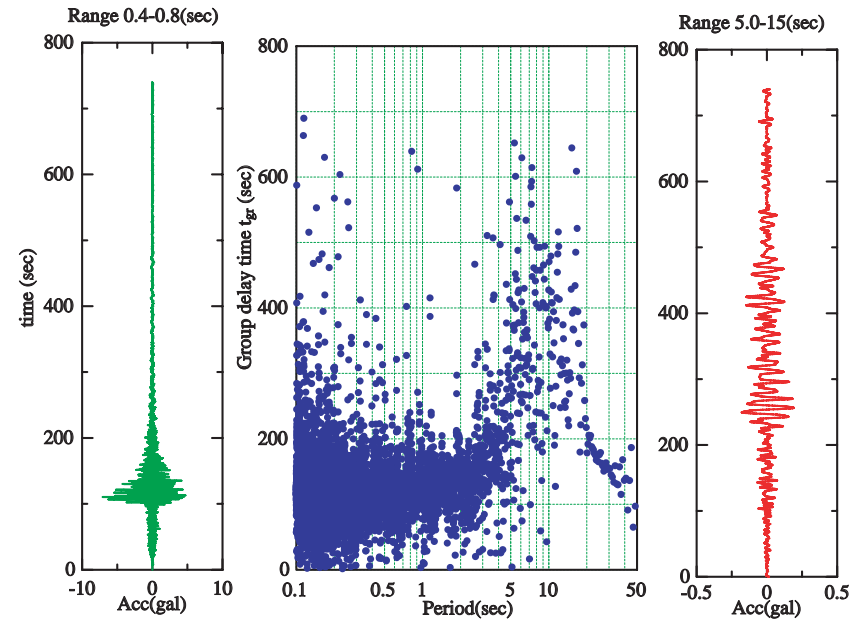

Fig. 1 Example of group delay time for earthquake ground motion

wave component with frequency $\omega$. The distribution width of the group delay time is related to the duration of the wave component's time history. These characteristics of group delay time make it much easier to model than directly modeling the phase spectrum. The mean group delay time $\mu_{t g r}(\omega)$ and its standard deviation $\sigma_{t g r}(\omega)$ are chosen as the characteristic parameters to represent the stochastic characteristics of the group delay time.

\subsection{Stochastic characteristics of group delay time}

We assume that the stochastic characteristics of the group delay time at a frequency of $\omega$ are Gaussian in nature. The characteristics are defined by its mean and covariance along the frequency. If the number of discrete sampling points of the group delay time is $N$ in a particular frequency domain, the probability density function of the discrete group delay time up to the circular frequency $\omega_{k}$ is expressed by 


$$
\begin{aligned}
& p\left(\Delta \phi^{(k)}\right)=\frac{1}{(2 \pi)^{\frac{j}{2}}\left|\mathbf{S}^{(k)}\right|^{\frac{1}{2}}} \\
& \times \exp \left\{-\frac{1}{2}\left(\Delta \phi^{(k)}-\mu_{t g r}^{(k)}\right)^{T} \mathbf{S}^{(j)-1}\left(\Delta \phi^{(k)}-\mu_{t g r}^{(k)}\right)\right\}
\end{aligned}
$$

where $\Delta \phi^{(k)}=\left(\Delta \phi_{1}, \Delta \phi_{2}, \cdots, \Delta \phi_{k}\right)^{T}$ and $\mu^{(k)}=\left(\mu_{t g r, 1}, \mu_{t g r, 2}, \cdots \mu_{t g r, k},\right)^{T} . \Delta \phi_{k}$ is the phase difference at the circular frequency $\omega_{k}$, defined by $\Delta \phi_{k}=t_{g r}\left(\omega_{k}\right) \cdot \Delta \omega . \mu_{t g r, k}$ is the average group delay time at the circular frequency $\omega_{k}$, while $\mathbf{S}$ is its covariance matrix. Since the correlation characteristics of the group delay time among neighboring circular frequencies has not yet been clarified, we neglected the correlation in this paper, and the group delay times were treated as being independent of each other along the frequency. In this case, the density distribution function is finally expressed by

$$
p\left(\Delta \phi^{(k)}\right)=\frac{1}{\sqrt{2 \pi} \sigma_{t g r}^{(k)}} \exp \left[-\frac{\left(\Delta \phi^{(k)}-\mu_{t g r}^{(k)}\right)^{2}}{2 \sigma^{(k) 2}}\right]
$$

where $\sigma_{t g r}^{(k)}=\left(\sigma_{t g r, 1}, \sigma_{t g r, 2}, \cdots ; \sigma_{t g r, k}\right)^{T} . \sigma_{t g r, k}$ is the standard deviation of the group delay time at the circular frequency $\omega_{k}$. Figure 2 shows a comparison between the normal distribution (i.e. the Gaussian distribution) function and the distribution of the calculated group delay time values. The normal distribution expresses the probabilistic characteristics of the group delay time well.

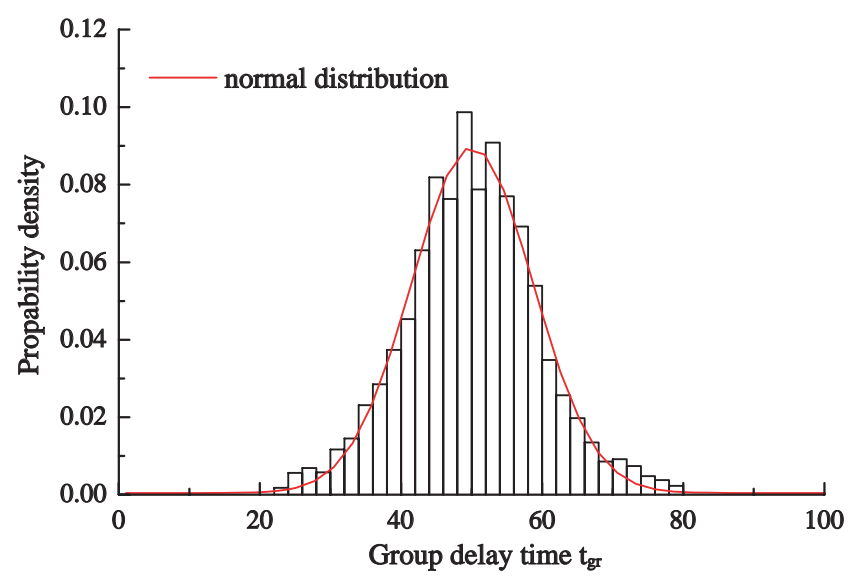

Fig. 2 Probability density of group delay time compared with the normal distribution function

\subsection{Mean and variance of group delay time}

A simple model has already been developed to derive the mean and variance of the group delay [2]. Using existing data sets from observed earthquakes, the group delay time of each earthquake motion was calculated for each frequency band range. If the phase spectrum $\phi^{(j)}\left(\omega_{k}\right)$ at a circular frequency of $\omega_{k}$ for the $j$-th frequency band is given, the group delay time is defined by

$$
t_{g r}^{(j)}=\frac{d \phi^{(j)}}{d \omega}=-\frac{\phi^{(j)}\left(\omega_{i}\right)-\phi^{(j)}\left(\omega_{i+1}\right)}{\Delta \omega}
$$

Due to the fluctuating nature of the group delay time in each frequency band, we calculate its average value $\mu_{t g r}^{(j)}$ and standard deviation $\sigma_{t g r}^{(j)}$ by the following equations:

$$
\mu_{t g r}^{(j)}=\sum_{i=1}^{N^{(j)}} \frac{t_{g r}^{(j)}\left(\omega_{i}\right)}{N^{(j)}}, \quad \sigma_{t g r}^{(j)}=\sqrt{\frac{1}{N^{(j)}} \sum_{i=1}^{N^{(j)}}\left(t_{g r}^{(j)}\left(\omega_{i}\right)-\mu_{t g r}^{(j)}\right)^{2}}
$$

Regression equations as functions of epicentral distance $R$ and earthquake magnitude $M$ for these values were derived [2]:

$$
\begin{aligned}
& \mu_{t g r}^{(j)}=\alpha_{1}^{(j)} \times 10^{\beta_{1}^{(j) M}} \times R^{\gamma_{1}^{(j)}} \\
& \sigma_{t g r}^{(j)}=\alpha_{2}^{(j)} \times 10^{\beta_{2}^{(j) M}} \times R^{\gamma_{2}^{(j)}}
\end{aligned}
$$

where $\alpha, \beta$ and $\gamma$ are regression coefficients. The superscript $j$ denotes a frequency band range given by $\left\{2^{j} / 3 T_{d} \leq f \leq 2^{j+2} / 3 T_{d}\right\}$, where $T_{d}$ is the duration time of the earthquake motion.

\section{Formulation of structural response considering the probabilistic phase spectrum}

\subsection{Response of single-degree-of-freedom system}

The time history of a non-stationary excitation $\ddot{z}(t)$ such as earthquake acceleration can be expressed by

$$
\ddot{z}(t)=\sum_{k=1}^{N} \ddot{z}_{k}(t)=\sum_{k=1}^{N} a_{k} \cdot \cos \left(\omega_{k} t+\phi_{k}\right)
$$

where $a_{k}$ and $\phi_{k}$ are the amplitude and phase of the $k$-th decomposed wave $\ddot{z}_{k}(t)$ at a discrete circular frequency $\omega_{k}$, respectively. The phase $\phi_{k}$ is defined by

$$
\phi_{k}=\phi_{0}+\sum_{l=1}^{k} \Delta \phi_{k}
$$

where $\phi_{0}$ is the initial phase, i.e. the phase at $\omega=0$.

The equilibrium equation of motion for a SDOF system for the decomposed wave $\ddot{z}_{k}(t)$ is given by

$$
\ddot{y}_{k}+2 h \omega_{0} \dot{y}_{k}+\omega_{0}^{2} y_{k}=-a_{k} \cos \left(\omega_{k} t+\phi_{k}\right)
$$

where $h$ is the damping constant and $\omega_{0}$ is the natural circular frequency of the SDOF system. The solution of this equation is obtained by the sum of the particular solution (i.e. the solution for stationary response) and the general solution (i.e. the solution for free vibration), as given by

$$
\begin{aligned}
& y(t)=\sum_{k=1}^{N_{f}} y_{k}(t)=\sum_{k=1}^{N_{f}} \Re\left[\left|C_{k}\right| \cdot\left(e^{i \omega k t}+e^{-h \omega_{0} t}\left(A_{k 1}+B_{k 1}\right) \cdot e^{i\left(\phi_{k}+\phi_{k}^{\prime}\right)}\right)\right] \\
& =\sum_{k=1}^{N_{f}} D_{k}(t) \cdot e^{i \phi_{k}^{\prime}} \\
& A_{k 1}=\frac{1}{2 \omega_{0} \sqrt{1-h^{2}}}\left(i h \omega_{0}-\omega_{k}-\omega_{0} \sqrt{1-h^{2}}\right) \cdot e^{i \omega^{\prime} t} \\
& B_{k 1}=-\frac{1}{2 \omega_{0} \sqrt{1-h^{2}}}\left(i h \omega_{0}-\omega_{k}+\omega_{0} \sqrt{1-h^{2}}\right) \cdot e^{-i \omega^{\prime} t}
\end{aligned}
$$

$C_{k}$ is the well known frequency response function of the SDOF system, and $\phi_{k}$ is the phase difference between the response and input motion, which is defined as follows:

$$
\begin{aligned}
C_{k} & =\left|\frac{-a_{k}}{\sqrt{\left(\omega_{0}^{2}-\omega_{k}^{2}\right)^{2}-\left(2 h \omega_{0} \omega_{k}\right)^{2}}}\right| \\
\phi_{k}^{\prime} & =\tan ^{-1}\left(-\frac{2 h \omega_{0} \omega_{k}}{\omega_{0}^{2}-\omega_{k}^{2}}\right)
\end{aligned}
$$


The velocity and acceleration responses are obtained from the derivative of $y(t)$ given by Eq. (10). The mean value of displacement for the $k$-th component, $y_{k}(t)$, is derived from

$$
E\left[y_{k}(t)\right]=\int_{-\infty}^{\infty} y_{k}(t) \cdot p\left(\phi_{k}\right) d \phi_{k}=\exp \left(i \phi_{0}\right) \cdot D_{k}(t) \cdot B_{k}
$$

Finally, the mean value of displacement $E[y(t)]$ is obtained from Eq. (12).

$$
E\left[y_{k}(t)\right]=\Re\left[\exp \left(i \phi_{0}\right) \sum_{k=1}^{N_{f}} D_{k}(t) \cdot B_{k}\right]
$$

When the initial phase $\phi_{0}$ is a uniform random value, the mean value becomes $E[y(t)]=0$. The covariance of response displacement $\operatorname{Var}[y(t)]$ is defined by

$$
\operatorname{Var}[y(t)]=E\left[y(t)^{2}\right]-(E[y(t)])^{2}
$$

This definition is finally expressed as

$$
\begin{aligned}
& \operatorname{Var}[y(t)]=\frac{1}{2} \sum_{k=1}^{N_{f}} D_{k} D_{k}^{\cdot}+\sum_{k=1}^{N_{f}-1} \sum_{j=k+1}^{N_{f}} D_{k} D_{j}^{\cdot} \cdot \frac{\left(\prod_{m=1}^{j} B_{m}\right)}{\left(\prod_{m=1}^{j} B_{k}\right)} \\
& +\Re\left\{\frac{1}{2} \cdot e^{2 i \phi_{0}} \sum_{k=1}^{N_{f}} A_{k} D_{k}^{2}+e^{2 i \phi_{0}} \sum_{k=1}^{N_{f}-1} \sum_{j=k+1}^{N_{f}} D_{k} D_{j} \cdot A_{j} \cdot \frac{\left(\prod_{m=1}^{j} B_{m}\right)}{\left(\prod_{m=1}^{j} B_{k}\right)}\right\} \\
& \{E[y(t)]\}^{2}
\end{aligned}
$$

When the initial phase $\phi_{0}$ is a uniform random value, the third and fourth terms on the right-hand side of Eq. (15) become zero.

\section{Numerical examples}

\subsection{Numerical conditions}

We demonstrate numerical examples calculated using our theoretical formulations as outlined above. The numerical conditions are listed below.

\section{A: Conditions for input motion}

1) Discrete sampling time: $\Delta t=0.02(\mathrm{sec})$

2) Number of data: $\mathrm{N}=1024$

3) Mean value of group delay time at each circular frequency: $\mu_{1}=\mu_{2}=\cdots=\mu_{N_{f}}=5$

4) Standard deviation of group delay time at each circular frequency: $\sigma_{1}=\sigma_{2}=\cdots=\sigma_{N_{f}}=2$

\section{B: Conditions for SDOF system}

1) Natural circular frequency of system: $\omega_{0}=6.28$

2) Damping constant of system: $h=5 \%$

\subsection{Comparison of theoretical solutions with sample response}

We can simulate sample input motions based on the following steps:

(i) Decide $\mu_{t g r}^{j}(\omega)$ and $\sigma_{t g r}^{j}(\omega)$ for input motion.

(ii) Generate random numbers based on normal distribution $N\left(\mu_{t g r}^{j}, \sigma_{t g r}^{j}\right)$ and calculate $t_{g r}^{j}(\omega)$. Obtain a phase spectrum $\phi_{j}(\omega)$ by integrating this with respect to $\omega$.
(iii)Simulate a time history $\ddot{z}(t)$ taking the inverse Fourier transform of the simulated phase spectrum $\phi(\omega)$ and amplitude spectrum $F(\omega)$.

A number of sample waves with similar stochastic characteristics can be simulated by changing the set of random numbers (see step (ii) above). The time histories of response velocity for the sample waves are calculated by solving the equilibrium equation of motion for an SDOF system. The Newmark $\beta$ method is employed for this purpose.

These sample response time histories are compared with the average wave $E[\dot{y}(t)]$ and the RMS $\sqrt{E\left[\dot{y}(t)^{2}\right]}$ wave previously described. We can see from Fig. 3 that RMS time functions are similar to the envelope of sample waves. In other words, the RMS represents the expectation of the envelope functions.
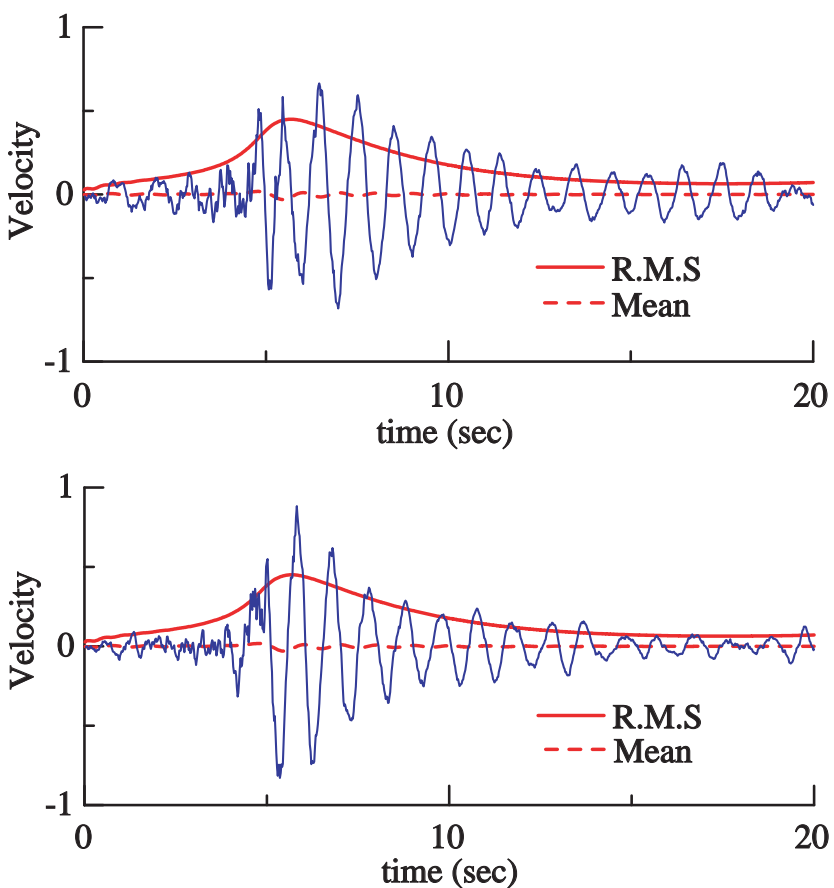

Fig. 3 Average wave and RMS wave compared with responses for sample waves

\subsection{Effects of initial phase on average wave $E[\dot{y}(t)]$ and RMS wave $\sqrt{E\left[\dot{y}(t)^{2}\right]}$}

We varied the initial phase $\phi_{0}$ to $\phi_{0}=0,0.25 \pi, 0.5 \pi$, and calculated the mean time function $E[\dot{y}(t)]$ and RMS time function $\sqrt{E\left[\dot{y}(t)^{2}\right]}$. Figure 4 shows the calculated results.

In the mean function $E[\dot{y}(t)]$, the influence of the initial phase appears at the time $t=\mu_{t g r}$. When the initial phase $\phi_{0}=0.5 \pi$, the mean response $E[\dot{y}(t)]$ is 0 . This suggests that the wave shape shows symmetry for $\phi_{0}=0.5 \pi$. When the initial phase is 0 , the mean response function changes significantly at the time $t=\mu_{t g r}$, showing that the shape of the response wave becomes non-symmetric. On the other hand, the RMS response function is less influenced by the initial phase. The envelope of response is independent of the initial phase. 


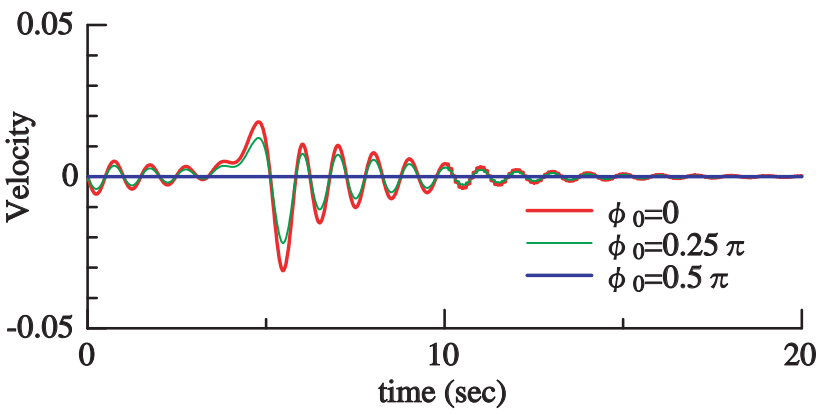

(a) average wave $E[\dot{y}(t)]$

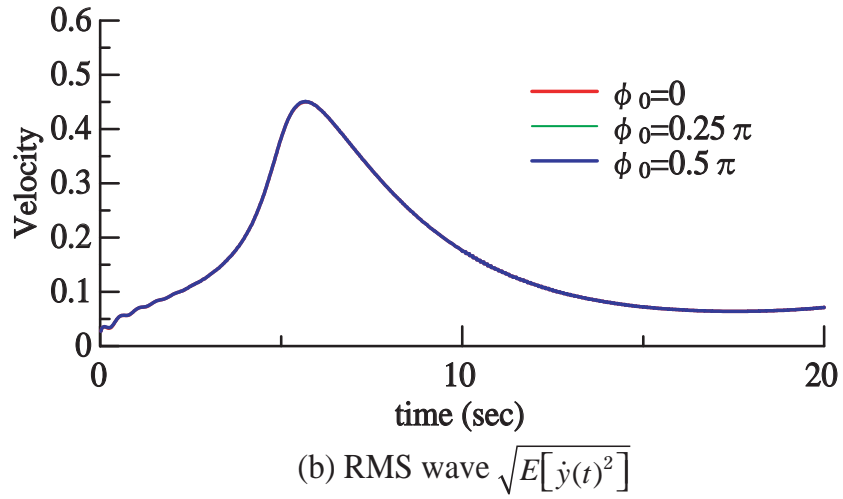

Fig. 4 Effects of initial phase on average wave $E[\dot{y}(t)]$ and RMS wave $\sqrt{E\left[\dot{y}(t)^{2}\right]}$

\section{Estimation of peak response value of SDOF system}

The response time history $y(t)$ will satisfy a causality condition. The Complex Envelope $y_{c}(t)$ has been defined [4]:

$$
y_{c}(t)=y(t)+i \cdot y_{H}(t)=Y(t) \cdot \exp (i \varphi(t))
$$

where $y_{H}(t)$ is the Hilbert transform of $y(t) . \quad Y(t)$ is the amplitude of the complex envelope, which represents the envelope of $y(t)$. As the shape of the root mean square $E_{\phi}\left[y^{2}(t)\right]$ of $y(t)$ is similar to the envelope of $y(t)$, the following assumption is introduced:

$$
y(t)=\alpha \cdot E_{\phi}\left\lfloor y(t)^{2}\right\rfloor
$$

The relationship between $Y(t)$ and $y(t)$ can be obtained by using Pareseval's theorem.

$$
\int_{-\infty}^{\infty} Y^{2}(t) d t=2 \times \int_{-\infty}^{\infty} y^{2}(t) d t
$$

The coefficient $\alpha$ is therefore determined by

$$
\int_{-\infty}^{\infty} Y^{2}(t) d t=\alpha^{2} \cdot \int_{-\infty}^{\infty} E_{\phi}\left[y^{2}(t)\right] d t=2 \times \int_{-\infty}^{\infty} y^{2}(t) d t
$$

The right side of Eq. (19) is readily calculated by the Fourier spectrum of input motion and the frequency response function using Pareseval's theorem.

A response spectrum can be easily calculated using this technique. Figure 5 shows examples of a velocity response spectrum. The coefficient $\alpha$ is about 2.0 for the whole range of the period. The response spectrum calculated by the technique outlined here is consistent with the results of the Monte Carlo simulation. This newly proposed method enables efficient estimation of the response spectrum in consideration of the stochastic characteristics of phase spectrum.

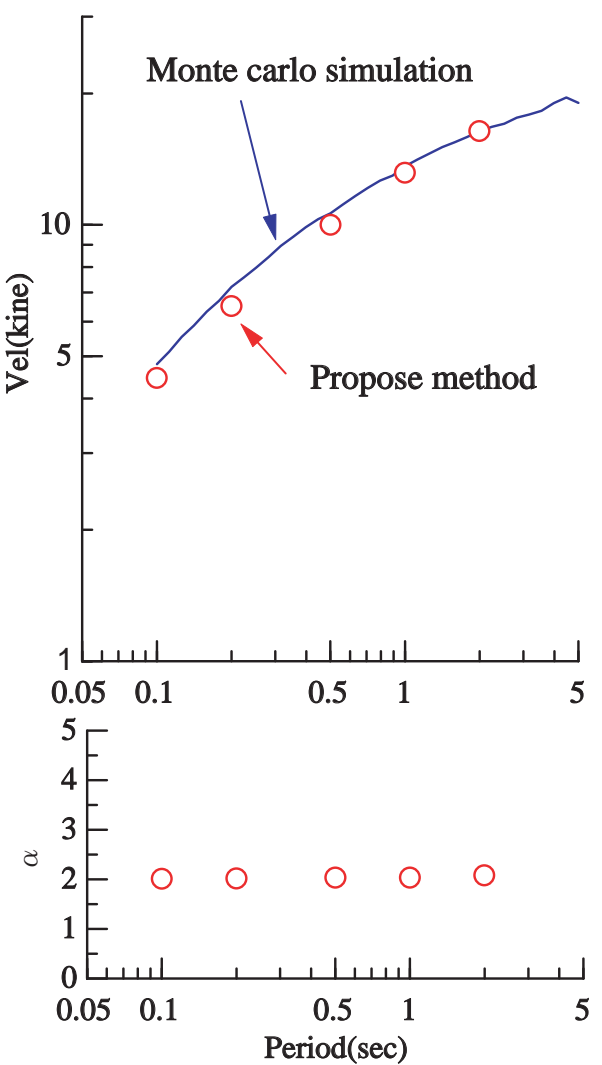

Fig. 5 Estimated results of velocity response spectrum compared with Monte Carlo simulation technique

\section{Conclusions}

We formulated the mean and variance of the structural response time history taking into account the probabilistic nature of the phase spectrum, and used this formulation to investigate the effects of the stochastic characteristics of phase spectrum on the time history of structural response. As a result, the response spectrum can be calculated easily only in frequency domain without using the time domain Monte Carlo Simulation.

\section{References}

[1] Kiureghian, A. D., "A Response Spectrum Method for Random Vibration Analysis of MDF Systems," Earthquake Engineering and Structural Dynamics, Vol.9, pp.419-435, 1981.

[2] Sato, T., Murono, Y. and Nishimura, A., "Modeling of Phase Spectrum to Simulate Design Earthquake Motion," in Elliott, W.M. and McDonough, P., Editors. Optimizing Post-Earthquake Lifeline System Reliability. Proceedings of the 5th US Conference on Lifeline Earthquake Engineering, Seattle, ASCE, 1999: pp.804-813, 1999.

[3] Papoulis, A., "The Fourier Integral and its Application," McGraw-Hill, 1962.

[4] Farnbach, J. S., "The Complex Envelope in Seismic Signal Analysis," Bulletin of the Seismological Society of America, Vol.65, No.4, pp.951-962, 1975. 